\title{
A New Wind Vector Retrieval Algorithm for C-band Airborne SAR
}

\author{
Yi Kong, Wenjun Liu*, Xianbin Zhao, Xi Chen, Hao An, Heng Bai \\ College of Meteorology and Oceanography \\ PLA University of Science and Technology \\ Nanjing, China \\ *liuwenjun106@gmail.coml
}

\begin{abstract}
A new ocean wind vector retrieval method is proposed that the wind speed and direction are estimated simultaneously using the normalized radar cross sections detected from different angles and geophysical model function according to the sounding characteristics of airborne SAR. The wind direction ambiguity is removed by using the odd symmetry property of polarimetric correlation coefficient with the changes in wind direction. To evaluate the ocean wind field retrieval errors and effects, the $C$ band airborne SAR data are used to obtain the wind speed and direction by the proposed method. Estimated wind vector are compared with wind measurements from buoys. We show that the wind field retrieval method is suited to retrieve high precision wind speed and direction from airborne SAR sounding data without other background sources.
\end{abstract}

Keywords-Retrieval; airborne synthetic aperture radar; wind vector; PPC

\section{INTRODUCTION}

Ocean wind field is the wind stress acted on the ocean, influences the interactions between the atmosphere and ocean circulation, also it is the key parameter in the study of the waves, ocean circulation, and marine meteorological, and airsea interaction. In marine applications, the sea surface wind field has an important impact on maritime navigation, marine engineering, marine fisheries monitoring, marine environmental pollution, as well as near-shore wind energy development and other human activities. Sea surface wind field detection has two ways: conventional detection and remote sensing. Tracking ships, buoys and other conventional direct detection method have high precision, but receive sparse data. Scatterometer, radiometer and other remote sensing devices can provide global wind vector information with resolution of $12.5 \sim 25 \mathrm{~km}$, but in the semi-enclosed bay, the sea ice edge strip and near-shore area, the detection value is vulnerable to the impact of land and sea-ice, which leads larger error of wind vector retrieval ${ }^{[1]}$.

Because of the high spatial resolution, Synthetic aperture radar (SAR), which can provide the details of the wind field of ocean and coastal areas, is an important remote sensing means of the sea surface wind field. The main platform equipped with SAR is the satellite. Subjected to the restrictions of the track, spaceborne SAR cannot meet the detection demand at the fixed time and location, so airborne SAR probe came into being. It plays an irreplaceable role in the fine detection of regional sea surface wind field. Currently, Spaceborne C-band SAR uses two wind vector retrieval methods. Firstly, using the technical means to obtain wind direction information, then retrieve wind velocity with CMOD series geophysical model function of $\mathrm{C}$ band VV polarization ${ }^{[2]}$. SAR wind direction algorithm is mainly based on wind streaks, including Fourier transform method $^{[3]}$, the local gradient method ${ }^{[4]}$, wavelet analysis ${ }^{[5]}$, or using the detection region's numerical forecasting model data or background wind direction of scatterometer data as input wind direction ${ }^{[6]}$. Secondly, observed field is provide by SAR data, the detection area's wind vector adopts numerical weather prediction model data or scatterometer data, then using the optimization algorithm to retrieve wind vector. The above two methods take advantage of auxiliary information in the retrieval wind vector. Owing to the CMOD series geophysical model function which contains at least two unknown geophysical parameters that is the wind speed and direction, the above two methods use auxiliary information in the retrieval of the wind vector, to modulated radar backscatter cross-section. Among them, the wind velocity and backward scattering cross section is shown as the exponential relationship, the wind direction and backward scattering cross section is shown as harmonic relationship. Therefore, to retrieve sea surface wind vector, a piece of SAR image or a group of backward scattering cross section and angle of incidence is not enough.

Restricted by the platform flight height of the airborne SAR, the probe image detection area ranges from a few kilometers to a dozen kilometers. Spaceborne SAR images whose detection range is a few hundred kilometers have wind streaks information which exists about $44 \%$, airborne SAR images are less likely to have wind streaks, moreover the wind direction derived from SWDA method also has the problem of $180^{\circ}$ ambiguity which need to be removed with external data ${ }^{[7]}$. That is why SWDA method is very difficult in the airborne SAR operational applications. In addition, airborne SAR has the characteristics of high mobility but limited by detection range in space and time, which means it is difficult to match the data of NWP and scatterometer detection. So NWP or scatterometer data could not directly be input to CMOD geophysical model 
function to calculate the wind velocity, and optimization algorithm can't be used to retrieve sea surface wind vector. Above all, how to use SAR detection data to retrieve wind vectors without the help of auxiliary data is an important issue of the Airborne SAR sea surface wind vector retrieval research.

Airborne SAR with the characteristics of high mobility can achieve continuous detection of the specific area. The wind vector of the region can usually be considered as basically consistent in a short time. Therefore, by means of route design, the retrieval program of Airborne SAR can be formed on the same observation ocean area.

\section{NEW SCHEME FOR AIRBORNE SAR SEA SURFACE WIND VECTOR RETRIEVAL}

\section{A. scheme research}

Via different course of flights in a short period of time, the Airborne SAR realizes the detection of the same water with several antennas which has different angles. Learned from foreign three antenna design experience of the scatterometer SCAT, we have designed approximate isosceles right-angle triangle route flight for carrier aircraft, making the gap of the detection angles approximately an integer multiple of $45^{\circ}$. Three sets of the probe data acquired from above means present minimum correlation, which is conducive to the cost function to solve optimal wind vector. Flight scheme is shown in Figure 1.

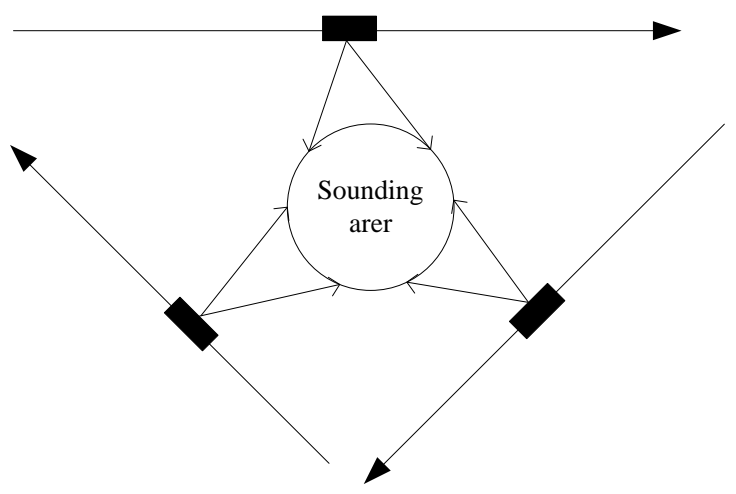

Fig. 1. The Airborne SAR flight program

Through the flight scheme, we can get the same angle of incidence in the same region. Corresponded to three azimuth angles $\phi_{1}^{a}, \phi_{2}^{a}$ and $\phi_{3}^{a}$, three radar backscattering cross section constitute three sets of observations for a cost function to calculate the wind vector.

In this case, the cost function is rewritten as:

$$
\begin{aligned}
J= & \left(\sigma_{1}^{r}-\sigma_{1}^{f}\left(u, \Phi-\phi_{1}^{a}, \theta\right)\right)^{2}+ \\
& \left(\sigma_{2}^{r}-\sigma_{2}^{f}\left(u, \Phi-\phi_{2}^{a}, \theta\right)\right)^{2}+\left(\sigma_{3}^{r}-\sigma_{3}^{f}\left(u, \Phi-\phi_{3}^{a}, \theta\right)\right)^{2}
\end{aligned}
$$

Where $\sigma^{r}$ is the real sounding $\mathrm{VV}$ polarization backscattering cross section of airborne $\mathrm{SAR}, \sigma^{f}$ is backscattering cross section derived from the CMOD5 geophysical model function simulation, $\Phi-\phi^{a}$ is the angle between the wind direction and the radar azimuth $\phi^{a}$. When it comes to detection area with a triangular flight, wind velocity $u$, wind direction $\Phi$ and angle of incidence $\theta$ can be treated as fixed value.

To solve the formula (1), the partial derivative of the cost functions on $u$ and $\cos (\Phi)$ must be equal to 0 .

$$
\begin{gathered}
\frac{\partial J}{\partial u}=0 \\
\frac{\partial J}{\partial \cos (\Phi)}=0
\end{gathered}
$$

But in the formula (1), $\Phi$ are related with azimuth $\phi^{a}$, not only, there are the third and fourth order of $\cos (\Phi)$. Thus, it is very difficult to obtain the analytical solution of the twodimensional nonlinear equation. As wind velocity within the range of $0 \sim 30 \mathrm{~m} / \mathrm{s}$, wind direction within the range of $0 \sim 360^{\circ}$ (extract value in accordance with wind velocity every $0.1 \mathrm{~m} / \mathrm{s}$, wind direction every $0.1^{\circ}$ ), numerical method will be taken to solve cost function of wind vector. Choosing wind vector whose cost function is minimum as retrieval result.

The wind vector retrieved by this method also exists wind direction ambiguity. This paper adopts latest research of fullpolarization SAR. Using the odd symmetry property, and the correlation coefficient of $\mathrm{VV}$ and $\mathrm{VH}$ polarization channel as the wind direction changes, to remove the wind direction ambiguity $^{[8]}$.

\section{B. The polarimetric wind direction ambiguity removal method}

Different from traditional non polarized SAR, more scattering characteristics of observation target can be recorded by full-polarization SAR data. Using the latest research results $^{[8]}, \sigma_{V H}^{0}$ and $\sigma_{H V}^{0}$ are equally even symmetry relations with the relative wind. Experimental studies have shown the correlation coefficient between different polarization scattering measurements (PCC), such as the correlation coefficient between $S_{V V}$ and $S_{V H}$, which is denoted as $p_{V V V H}$.

$$
p_{V V V H}=\frac{<S_{V V} \cdot S_{V H}^{*}>}{\sqrt{<\left|S_{V V}\right|^{2}><\left|S_{V H}\right|^{2}>}}
$$

This is a plural to denote the Relevance between $S_{V V}$ and $S_{V H}$. By a large number of experiments, real and imaginary parts of polarization correlation function are odd symmetry on the wind direction. The symmetry can effectively remove the wind blur. Its rule is:

1. If the real part $<0$ and the imaginary part of $>0$, $0^{\circ}<\Phi<90^{\circ}$ 
2. If the real part $>0$ and the imaginary part of $>0$, $90^{\circ}<\Phi<180^{\circ}$

3. If the real part $<0$ and the imaginary part of $<0$, $180^{\circ}<\Phi<270^{\circ}$

4. If the real part $>0$ and the imaginary part of $<0$, $270^{\circ}<\Phi<360^{\circ}$

Therefore, by calculating the real part and the imaginary part of the polarization correlation coefficient, the problem of the wind direction ambiguity can be solved.

\section{The retrieval process}

Specific steps of ocean wind vector retrieval method are as follows:

1. The observation angle of incidence, the radar azimuth and three azimuth detection backscattering cross sections will be input to cost function which contains CMOD5 geophysical model function.

2. As wind velocity within the range of $0 \sim 30 \mathrm{~m} / \mathrm{s}$, wind direction within the range of $0 \sim 360^{\circ}$ (extract value in accordance with wind velocity every $0.1 \mathrm{~m} / \mathrm{s}$, wind direction every $0.1^{\circ}$ ), numerical method will be taken to solve cost function of each set of wind speed, wind direction. Choosing two sets of wind vector whose cost function is minimum as retrieval wind vector.

3. Calculating the VV and VH channel's correlation coefficient on first edge's detection data of the triangle route. Using odd symmetry property shown by polarimetric correlation coefficient as the wind direction changes, to remove the blur wind and get the only wind vector.

4. Comparing the retrieval results with buoy observations, verifying the effectiveness of the retrieval method, and analyzing the influence of airborne SAR technical parameters on retrieval result.

\section{FLIGHT SCHEME AND EXPERIMENTAL DATA}

\section{A. Flight scheme}

To further verify the inversion method proposed in the paper, we make use of airborne C-band full-polarization SAR to launch a probe test. Choosing $50 \mathrm{~km} \times 50 \mathrm{~km}$ area around the buoys as flight experiment and approximate isosceles rightangle triangle heading flight. To ensure the effectiveness of the detection, completion of a triangular flight time should be as short as possible, generally not more than 20 minutes, and each triangle use the same flight altitude, angle of incidence and resolution to form the effect that three azimuth antenna detect the same waters.

\section{B. Experimental data}

The experimental probe data is C-band full-polarization SAR data, including backward scattering cross section $\sigma_{H H}^{0}$, $\sigma_{H V}^{0}, \sigma_{V V}^{0}, \sigma_{V H}^{0}$, Paper use data including VV polarization backscattering cross section $\sigma_{V V}^{0}, \mathrm{VV}$ and $\mathrm{VH}$ polarization scattering coefficient $S_{V V}, S_{V H}$. Flight detection time of Airborne SAR is July 26, 2012. Buoy data provide wind vector at $10 \mathrm{~m}$ height over the sea, once every ten minutes, for the wind vector retrieval accuracy quantitative comparison.

\section{ANALYSIS OF RESULTS}

In order to verify the effectiveness for the proposed method in the paper, analyzing the retrieval results of sea surface wind vector quantitatively. In this section, 5 sets of experimental data on July 262012 will be processed, and compared with the actual results of buoy. The results obtained are shown below:

TABLE I. RESULTS OF WIND FIELD RETRIEVAL

\begin{tabular}{|c|c|c|c|c|}
\hline $\begin{array}{c}\text { Data } \\
\text { code }\end{array}$ & $\begin{array}{c}\text { Retrieval } \\
\text { wind } \\
\text { speed(m/s) }\end{array}$ & $\begin{array}{c}\text { Retrieval } \\
\text { wind } \\
\text { direction( }{ }^{\circ} \text { ) }\end{array}$ & $\begin{array}{c}\text { Buoy wind } \\
\text { speed(m/s) }\end{array}$ & $\begin{array}{c}\text { Buoy wind } \\
\text { direction( }{ }^{\circ} \text { ) }\end{array}$ \\
\hline 2601 & 6.3 & 131.9 & 5.8 & 134.0 \\
\hline 2602 & 6.5 & 138.6 & 5.5 & 143.0 \\
\hline 2603 & 7.2 & 140.5 & 6.5 & 146.0 \\
\hline 2604 & 5.4 & 133.9 & 6.5 & 146.0 \\
\hline 2605 & 6.5 & 144.3 & 5.9 & 140.0 \\
\hline RMS & \multicolumn{2}{|c|}{0.8} & \multicolumn{2}{c|}{6.6} \\
\hline
\end{tabular}

From figure 1 we can see that, the RMS of wind speed is $0.8 \mathrm{~m} / \mathrm{s}$, and the RMS of wind direction is $6.6^{\circ}$

\section{CONCLUSION AND PROSPECT}

Airborne SAR has the characteristics of high mobility, the paper puts forward a different wind vector retrieval method based on the different detection direction, which retrieves sea surface wind vector directly using airborne SAR the data without the aid of auxiliary information. Solving the problem that traditional spaceborne SAR sea wind vector retrieval method doesn't apply to the airborne platform. The paper conducts retrieval experiments of airborne full-polarization SAR probe data, verifies the effectiveness of the retrieval method.

Ocean surface wind vector retrieval method based on the different detection direction solve the problem of wind vector retrieval, polarization correlation coefficient accurately removes the wind ambiguity. The method uses the phase difference brought about by different detection directions to retrieve wind vector, and receives higher retrieval accuracy.

Results of the paper have important significance in operational detection of ocean surface wind vectors for Airborne SAR. Due to the limitations of the test conditions, the article only carried out three cruises detection test, and we will use the test data to verify the effectiveness of the retrieval method in the next step.

\section{REFERENCES}

[1] Martin, Seelye. An introduction to ocean remote sensing. Cambridge University Press, 2004. 
[2] Verhoef, Anton, et al. "CMOD5. n-the CMOD5 GMF for neutral winds." EUMETSAT Ocean and Sea Ice SAF Tech. Note SAF/OSI/CDOP/KNMI/TEC/TN/165 (2008).

[3] Vachon, P. W., and F. W. Dobson. "Validation of wind vector retrieval from ERS-1 SAR images over the ocean." The Global Atmosphere and Ocean System 5.2 (1996): 177-187..

[4] Koch, Wolfgang. "Directional analysis of SAR images aiming at wind direction." Geoscience and Remote Sensing, IEEE Transactions on 42.4 (2004): 702-710.

[5] Leite, Gladeston C., et al. "Wavelet analysis for wind fields estimation." Sensors 10.6 (2010): 5994-6016.
[6] Yang, Xiaofeng, et al. "Comparison of ocean-surface winds retrieved from QuikSCAT scatterometer and Radarsat-1 SAR in offshore waters of the US west coast." Geoscience and Remote Sensing Letters, IEEE 8.1 (2011): 163-167.

[7] Kong, Y., et al. "Ocean surface wind field retrieval from airborne SAR imagery based on Mexican-hat wavelet transform." Journal of PLA University of Science and Technology(Natural Science Edition)/Jiefangjun Ligong Daxue Xuebao 12.3 (2011).

[8] Zhang, Biao, et al. "Ocean Vector Winds Retrieval From CBand Fully Polarimetric SAR Measurements." (2012): 1-10. 